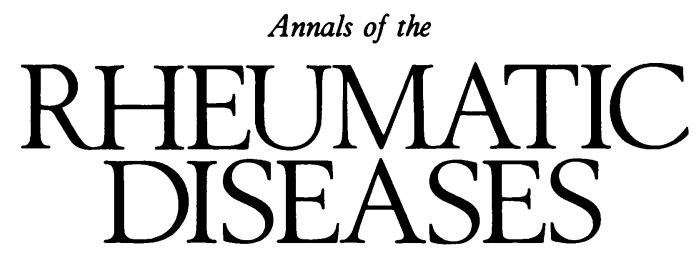

Leader

\title{
How serious is knee osteoarthritis?
}

Knee osteoarthritis is a major public health problem in the United Kingdom which presently receives relatively scant resources for treatment or clinical research. Most rheumatologists do not follow up patients with osteoarthritis once the diagnosis has been made and few have an active academic interest in osteoarthritis. There is often a fatalistic attitude towards outcome and prognosis throughout the health care professions. This brief review attempts to clarify three points: $(a)$ how common is the disease; $(b)$ what are its consequences in terms of symptoms and disability; and $(c)$ what proven treatments are available and which patients will benefit from intervention.

Quantifying the importance of knee osteoarthritis is not easy. It can be defined in a number of different ways using radiographs, reported symptoms, clinical signs, or loss of function leading to disability or handicap. For subjects over the age of 45 years most population surveys show that the prevalence of radiographically determined knee osteoarthritis ranges from 14 to $30 \%$ in men and women, rates being consistently higher in women and increasing steadily with age. ${ }^{1-8}$ If knee pain is used as a definition of osteoarthritis similar prevalence rates are found, with studies reporting between 12 and $38 \%$ of subjects over 45 years being affected. ${ }^{1-9}$ Most studies show that the proportion of radiological disease which is symptomatic is between $\mathbf{4 0}$ and $80 \%$, increasing with disease severity. ${ }^{78}$ Some work has suggested that the relation may be strengthened if patellofemoral views are also used. Prevalence rates also vary dramatically with how knee pain is categorised; using the new American College of Rheumatology (ACR) criteria for clinical osteoarthritis (which require that pain is current and present on most days in the previous month), one study showed that the prevalence of knee osteoarthritis decreased to less than $3 \%$ in middle aged subjects. ${ }^{10}$ The low sensitivity of the ACR criteria in normal populations also suggests that they are not appropriate for use in population studies and should be restricted to clinical trials of symptomatic patients. Most population studies have used a crude system of classifying a subject as symptomatic if knee pain was present for most days of any month in the past. Thus it appears that many people with radiological osteoarthritis may have intermittent or episodic knee pain.

Only scanty data are available on the likelihood of disability resulting from knee osteoarthritis. The NHANES I follow up study examined physical functioning in 2884 adults aged over 45 years, 10 years after the initial examination. Forty three per cent of women with knee osteoarthritis and $29 \%$ of men had difficulty walking a quarter of a mile.
Although reported disability increased with radiological severity and was greater in those with knee pain, disability was still increased in those with asymptomatic knee osteoarthritis. " Similar results were obtained in a different analysis of the same data set ${ }^{6}$ and are supported by cross sectional studies, which suggest that over $50 \%$ of men and women with symptomatic knee osteoarthritis reported some loss of function or disability.

If these prevalence rates are extrapolated to the current England and Wales population aged over 45 years, between 1.6 and 3.4 million subjects have symptomatic radiological knee osteoarthritis and between 900000 and 1.9 million of these will have associated disability. At present it is likely that only a small fraction of these are treated by specialists in the United Kingdom. If early intervention could prevent some of this disability a major effect on public health could be achieved. There are, however, a number of problems. The first is to determine which patients would benefit from intervention. It is obviously inappropriate to attempt to treat up to three million symptomatic patients in the United Kingdom without a clearer understanding of the course and prognosis of the disease.

The natural history of knee osteoarthritis is poorly understood. A few small longitudinal studies have indicated that between 30 and $50 \%$ of patients seen in a hospital clinic will continue to deteriorate, but many remain stable over many years. ${ }^{12-14}$ This is also suggested by cross sectional data in elderly subjects. ${ }^{15}$ Although traditionally associated with morbidity, osteoarthritis has also been shown to increase mortality. ${ }^{16}$ It is likely that comorbid factors are partially responsible for this, but it has also been suggested that there is a marked excess of deaths from gastrointestinal disease related to the use of non-steroidal anti-inflammatory drugs (NSAIDs) and analgesic drugs, though the attributable risks are unclear. ${ }^{17}$

The data available so far from clinical studies are too few to derive reliable prognostic indicators to select the group at highest risk. In population studies those with longstanding knee pain and poor psychological ratings do worse in terms of disability. ${ }^{611}$

The second dilemma is that if a high risk group could be reliably selected, are there any interventions which would be effective in the long term in reducing disability? Short term studies of treatment with NSAIDs have confirmed their effectiveness in terms of symptoms in many patients but no studies have addressed long term outcome, and their benefit relative to simple analgesic drugs is controversial. ${ }^{18}$ Similary, the long term benefits of physiotherapy and intra- 
articular injections have not been evaluated, though a controlled study of a supervised walking programme showed encouraging short term results with respect to function. ${ }^{19}$ Although advice on weight reduction is often given by dieticians or doctors, little is known about its effectiveness in patients with clinical osteoarthritis. ${ }^{20}$

At present the only proven long term treatment is total knee replacement. Results have now become as good as, or in some cases surpassed, those for replacement of the hip joint. ${ }^{21}$ Ten year survival rates are now reported to be $97 \%$, relieving pain in $95 \%$ and function in $90 \%$ of patients. ${ }^{22}$ If, however, surgical intervention is delayed and standard condylar prostheses cannot be used, results are poorer. ${ }^{23}$ In 1991 in England and Wales, according to the Department of Health, an estimated 12693 knee arthroplasties were performed. Most of these (over 60\%) were for osteoarthritis. This compares with an estimated 30000 total hip replacements despite the prevalence of knee osteoarthritis being two- to fivefold greater than osteoarthritis of the hip. A study from the Mayo Clinic suggests that in the USA there is an underutilisation of knee arthroplasty. ${ }^{24}$ If the current rates of knee arthroplasty performed in the Mayo Clinic were applicable to the United Kingdom, we should be performing over 36000 operations, three times the number of knee replacements each year than are actually carried out.

Although in terms of its effect on public health, osteoarthritis research is relatively underfunded in all aspects, priority should be given to practical issues in two main areas: funding long term clinical outcome trials of drug and non-drug treatments, and devising methods of selecting subjects with knee osteoarthritis most likely to become severely disabled and who would therefore benefit from early referral for knee joint replacement.

Department of Rheumatology,

StBartholomew's Hospital,

T DSPECTOR

Charterhouse Square,

London ECIM $6 B Q$

United Kingdom
1 Bagge E, Bjelle A, Eden S, Svanborg A. Osteoarthritis in the elderly. Ann Rheum Dis 1991; 50: 535-9.

2 Bergstrom G, Bjelle A, Sundh V, Svanborg A. Joint disorders at age 70, 75 and 79, a cross-sectional comparison. Br f Rheumatol 1986; 25: $333-41$.

Carman W J. Factors associated with pain and osteoarthritis in the Tecumseh Community Health Study. Semin Arthritis Rheum 1989; 18: 10-3.

4 Lawrence J S, Bremner J M, Bier F. Osteoarthritis: prevalence in the population and relation between symptoms and $x$ ray changes. Ann Rheum Dis 1966; 25: 1-23.

5 Classens A A M C, Shouten J S A G. Do clinical findings associate with radiographic osteoarthritis of the knee. Ann Rheum Dis 1990; 47: 771-4.

6 Hochberg M C, Lawrence R C, Everett D F, Cornoni-Huntley J. Epidemiologic association of pain in osteoarthritis of the knee: data from NHANES and NHANES I epidemiologic follow-up study. Semin Arthritis Rheum 1989; 18: 4-9.

7 Felson D T, Naimark A, Anderson J, Kazis J, Castelli W, Meenan R F. The prevalence of knee osteoarthritis in the elderly: the Framingham osteoprevalence of knee osteoarthritis in the elderly:
arthritis study. Arthritis Rheum 1987; 30:914-8.

8 Hart D J, Spector T D, Brown P, Wilson P, Doyle D V, Silman A J. Clinical signs of early osteoarthritis: reproducibility and $x$ ray changes in 541 women in the general population. Ann Rheum Dis 1991; 50: 467-70.

9 McAlindon T E, Cooper C, Kirwan J R, Dieppe PA. Knee pain and disability in the community. Br $\mathcal{Y}$ Rheumatol 1992; 31: 189-92.

10 Spector T D, Hart D J, Leedham-Green $M$. The prevalence of knee and hand osteoarthritis (OA) in the general population using different clinical criteria: the Chingford study [abstract]. Arthritis Rheum 1991; 34: 5171.

11 Davis M A, Ettinger W H, Nevhavs J M, Mallon K P. Knee osteoarthritis and physical functioning: evidence from the NHANES I epidemiologic followup study. F R heumatol 1991; 18: 591-8.

12 Hernborg J S, Nilsson B E. The natural course of untreated osteoarthritis of the knee. Clin Orthop 1977; 123: 130-7.

13 Massardo L, Watt I, Cushnaghan J, Dieppe P. Osteoarthritis of the knee joint: an eight year prospective study. Ann Rheum Dis 1989; 48: 893-7.

14 Spector T D, Dacre J E, Harris P A, Huskisson E C. The radiological progression of osteoarthritis; an 11 year follow up study of the knee. Ann Rheum Dis. In press.

15 Forman M D, Malamet R, Kaplan D. A survey of osteoarthritis of the knee in the elderly. F Rheumatol 1983; 10: 282-7.

16 Monson R R, Hay A P. Mortality among arthritis. $f$ Chronic Dis 1976; 29: 459-67.

17 Hochberg M C. Association of non-steroidal anti-inflammatory drugs with upper GI disease: epidemiologic and economic considerations. F Rheumatol. In press.

18 McAlindon B J R, Dieppe P A. The medical management of OA of the knee: an inflammatory issue. Brf Rheumatol 1990; 29: 471-3.

19 Kovar P A, Allegrante J P, Mackenzie C R, Peterson M G E, Gutin B, Charlson M E. Supervised fitness walking in patients with osteoarthritis of the knee. Ann Intern Med 1992; 116: 529-34.

20 Spector T D. The fat on the joint: obesity and OA. F Rheumatol 1990; 17: 283-4.

21 Quam J P, Michet C J, Wilson M G, et al. Total knee arthroplasty: a population based study. Mayo Clin Proc 1991; 66: 589-95.

22 Noble J, Hilton R C. Total knee replacement: getting better all the time. BMF 1991; 303: 262.

23 Ranawat C S, Boachie-Adjei O. Survivorship analysis and results of total condylar knee arthroplasty; 8-11 year follow-up period. Clin Orthop 1988; 226: 6-13.

24 Rand J A, Ilstrup D M. Survivorship analysis of total knee arthroplasty. f Bone foint Surg [Am] 1991; 73: 397-401 